\title{
An Experimental Characterization of Thermophysical Properties of a Porous Ceramic Shell Used in the Investment Casting Process
}

\author{
Jones, C. A.*; Jolly, M. R.*; Jarfors, A. E. W.**; Irwin, M.*** \\ c.a.jones@cranfield.ac.uk
}

ORCID ID: 0000-0003-4714-8531

\author{
*Sustainable Manufacturing System Centre, Cranfield University, Bedfordshire, MK43 0AL, United Kingdom \\ **Jönköping University, School of Engineering, Box 1026, 55111 Jönköping, Sweden \\ *** TPC Components AB, Brånstaleden 2, 73432 Hallstahammer, Sweden
}

\begin{abstract}
This study presents the results of an investigation that characterises the thermophysical properties of an investment casting mould, comprising of a zirconium dioxide/cobalt aluminate prime slurry and a fused silica/fibre reinforced backup slurry. Growing prevalence of successful computer simulations within the foundry industry enables defects that emerge during the casting process to become increasingly predictable, providing cost-effective alternatives to trial castings. The viability of these simulations as predictors is heavily dependent upon the facilitation of accurate material property data, as attained through this investigation. Differential scanning calorimetry (DSC) and laser flash analysis (LFA) were utilized to determine the specific heat capacity and thermal diffusivity respectively. These values, in combination with the material density and linear coefficient of thermal expansion, have been used to determine the thermal conductivity of the mould. With the aim of verifying these parameters, initial studies in Flow-3D ${ }^{\circledR}$ simulation software have been performed to determine the constraints needed to reduce variability in simulation parameters. Due to the diversity of casting moulds used throughout the industry, ensuring the material database is kept as comprehensively populated as possible is a crucial undertaking.
\end{abstract}

Keywords: Component casting, thermophysical properties, conductivity, porosity, CFD simulation, sustainable

\subsection{Introduction}

Investment casting is the preferred manufacturing technique for the production of high-quality components for aerospace applications as a result of the geometric complexity and dimensional tolerances it affords [1]. As computers become more powerful, the process optimizing the production of such complicated parts has gradually been moving from the traditional trial and error approach [2] utilized by foundries of old to much more resourceefficient computer simulations.

Any computer simulation is only as accurate as the physical parameters underpinning its predictive capabilities. A comprehensive understanding of mould thermophysical properties is crucial to obtaining any meaningful estimations of the cooling rate, temperature gradient, solidification time, microstructural development etc. Many previous papers are concerned with the investigation of investment casting moulds, targeting mechanical properties, such as strength, using techniques including modulus of rapture (MOR) [3] [4] as well as mould creep and permeability [5]. Literature containing a comprehensive collection of material property data, especially thermophysical data is still a rarity.

Previous research work conducted by [6] on identical mould material considered the impact of fluctuations in critical process parameters introduced as a result of equipment and human involvement. His work included material characterisation and assessment of how accurately mould filling can be predicted; however, the prediction of important solidification parameters, such as temperature gradient and cooling rate, were not assessed.

In this work, thermophysical properties have been experimentally determined for an industrial investment casting mould. The aim is to inform boundary conditions for computer simulations/verifications and, in future work, act as a basis for the prediction and statistical optimisation of microstructural development in cast components. 


\section{$\underline{\text { 2.0 Methodology }}$}

The shelling system considered in this analysis is employed during the casting of Ni-based superalloys, the compositional breakdown of which is presented in table 1 . The shell consists of a prime coat supported by multiple backup layers which, generally speaking, have a combination of Zirconia and fused silica reinforced with fibres.

\begin{tabular}{|c|c|c|c|}
\hline Coating & Composition & \multicolumn{2}{|c|}{ Details } \\
\hline \multirow{4}{*}{ Prime } & & $\mathrm{ZrO}_{2}$ & $>63.5 \%$ \\
& Chilches & $\mathrm{TiO}_{2}$ & $<0.2 \%$ \\
& Microzir Flour & $\mathrm{Fe}_{2} \mathrm{O}_{3}$ & $<0.15 \%$ \\
& $200 \mathrm{M}$ IC & $\mathrm{Al}_{2} \mathrm{O}_{3}$ & $<1.3 \%$ \\
& & $\mathrm{SiO}_{2}$ & $<33 \%$ \\
\cline { 2 - 4 } & Cobalt & $\mathrm{CoAl}_{2} \mathrm{O}_{4}$ & $100 \%$ \\
\cline { 2 - 4 } & Aluminate & \multicolumn{3}{|c|}{ Binder } \\
\cline { 2 - 4 } & Primecote Plus & \multicolumn{3}{|c|}{-} \\
\cline { 2 - 4 } & DCH-10 & \multicolumn{2}{|c|}{ Binder } \\
\hline \multirow{4}{*}{ Antifoam } & Rackup & \multicolumn{2}{|c|}{ Fibre } \\
\cline { 2 - 5 } & Matrixol 30 & $\mathrm{SiO}_{2}$ & $\approx 100 \%$ \\
\cline { 2 - 5 } & MXC Excel X2 & \multicolumn{2}{|c|}{$\mathrm{Reinforcement}^{2}$} \\
\cline { 2 - 5 } & DI Water & $\mathrm{H}_{2} \mathrm{O}$ & $100 \%$ \\
\hline
\end{tabular}

Table 1. Ceramic Mould Composition

\subsection{Laser Flash Analysis (LFA)}

A Netzsch LFA 427 instrument was used in connection with a TASC 414/4 measurement unit to determine the thermal diffusivity of the mould material.

A cylindrical sample with a diameter of $12.8 \mathrm{~mm}$ and a height of $5.1 \mathrm{~mm}$ was coated with a thin layer of Graphite and Chromium before being placed in the path of a 1064nm Helium laser, just ahead of a liquid Nitrogen cooled sensor array. The system was configured so that the sample would be heated at a rate of 50 Kelvin per minute to $1000^{\circ} \mathrm{C}$ before any laser shots were conducted. Five shots were taken at each chosen temperature before the temperature was reduced by $200^{\circ} \mathrm{C}$ between each group of shots.

\subsection{Pycnometry}

A Micromeritics AccuPyc 21340 Pycnometer, configured to use Nitrogen gas as opposed to Helium, was used to measure the density of the shell material as well as providing information on the shell porosity. A cylindrical sample was placed in the testing sleeve and the computer system, operating a virtual 32-bit operating system, overseeing the equipment operation was programmed to undertake 15 repeat cycles to minimise the standard errors.

\subsection{Differential Scanning Calorimetry (DSC)}

A Netzsch 404 Pegasus DSC instrument was used to determine the heat capacity of the ceramic mould. Samples of mould were taken from three locations - prime coat, the approximate centre and the outer most surface - for analysis with masses $40.4 \mathrm{mg}, 41.4 \mathrm{mg}$ and $40.3 \mathrm{mg}$ respectively. These masses are as close to $40 \mathrm{mg}$ as possible to ensure they are comparable with the Sapphire baseline required to calculate the heat capacity.

These samples were placed individually into the testing crucible and sealed in the chamber before the atmosphere was flooded with Argon. The temperature cycle was selected to commence at $25^{\circ} \mathrm{C}$ and rise to $1400^{\circ} \mathrm{C}$ before returning to $25^{\circ} \mathrm{C}$ at a rate of 10 Kelvin per minute.

\subsection{Dilatometry (DiL)}

A Netzsch 402 DIL instrument was utilized to determine the coefficient of thermal expansion (CTE) of an approximately cylindrical sample of mould material. Like the DSC, these samples must be compared to a reference sample to evaluate the CTE; a fact that limits the sample dimensions to either $12 \mathrm{~mm}$ or $25 \mathrm{~mm}$ in length with a diameter of approximately $6 \mathrm{~mm}$. 
One sample of each length has been tested to allow compares of any differences between the two lengths. Individually, these samples are sealed into the experimental chamber and immersed in a Helium atmosphere. An identical temperature cycle was used in both cases in which the temperature increases to $1400^{\circ} \mathrm{C}$ from the initial $25^{\circ} \mathrm{C}$ before returning to $25^{\circ} \mathrm{C}$ at a rate of 10 Kelvin per minute.

\section{$\underline{\text { 3.0 Results and Discussion }}$}

\subsection{Laser Flash Analysis (LFA)}

The mould sample that was coated solely with Graphite was not able to produce any usable results, even follow multiple attempts. Such an outcome suggests that the Graphite-mould combination has significant transparency to the laser preventing the acquisition of data. Previous work has exploited such transparency at certain frequencies to monitor the filling of the mould cavity in real-time [7].

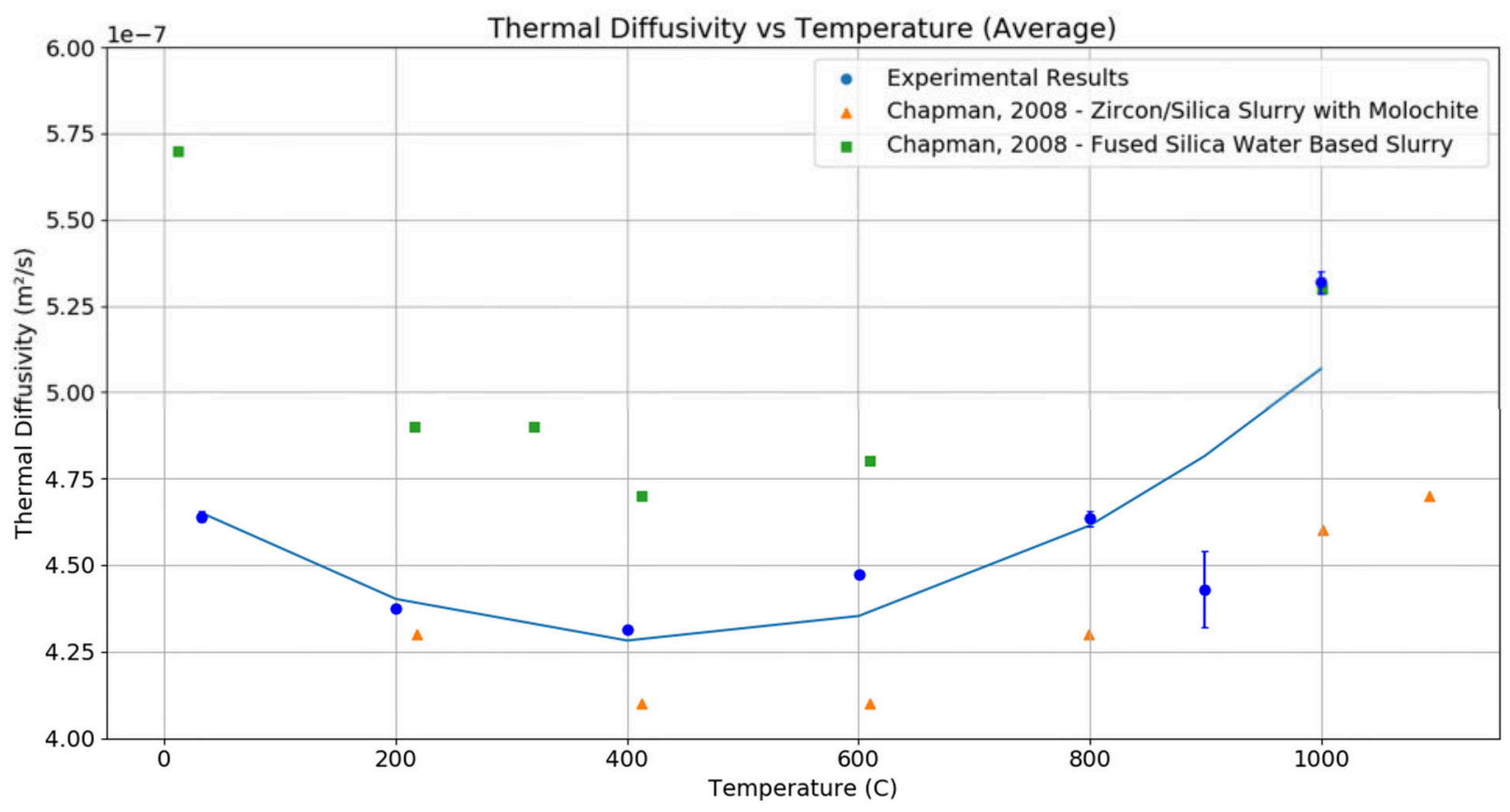

Figure 1. Comparative plot between the experimental thermal diffusivity profile and results published in literature as a function of temperature

A marked improvement in results was observed by coating the sample with a combination of Graphite and Chromium; the resulting thermal diffusivity measurements are illustrated in figure 1 alongside published experimental data from another relevant study [8]. The experimental data is in reasonable agreement with the published data laying between the two available sets with an average value of $4.64 \times 10^{-7} \mathrm{~m}^{2} / \mathrm{s}$. The data point seen at $850^{\circ} \mathrm{C}$ is the result of an unintended shot made by the computer system overseeing the instrument's operation. Only one shot was taken at this point as opposed to the five taken at all other points and as a consequence, the associated error is significant. A second degree least squares polynomial fitting function has been applied to the data to illustrate the data profile as a function of temperature, which takes the form as seen in equation 1.

$$
\alpha(T)=2.39 \times 10^{-13} T^{2}-2.03 \times 10^{-10} T+4.71 \times 10^{-7}
$$

It was intended to attain thermal diffusivity results for temperatures up to and including $1500^{\circ} \mathrm{C}$, however even with the Chromium coating it was not possible to gather data above $1000^{\circ} \mathrm{C}$. It might be possible to solve this issue by using powdered shell samples in place of the intact samples; a technique that can be employed when using LFA equipment.

\subsection{Differential Scanning Calorimetry (DSC)}

DSC results were obtained independently for each of the three samples taken from the shell specimen, representing different regions within a typical shell. To estimate the overall heat capacity of the shell, a rule of mixtures 
approach (equation 2) was used which has been presented in the published literature as an appropriate technique when considering ceramics materials [9].

$$
C_{p}^{e f f}=\sum_{i}\left(\frac{A_{i} M_{i}}{A_{1} M_{1}+A_{2} M_{2}+A_{3} M_{3}}\right) C_{p}^{i}
$$

Here $C_{p}^{e f f}$ is the effective specific heat capacity, $i$ is an integer ranging in value from 1 to 3 denoting the innermost sample, centre sample and the outer most sample respectively, $A_{i}$ is a mass dependent scaling coefficient, $M_{i}$ is the sample mass and $C_{p}^{i}$ is the specific heat capacity.

An important consideration in the scaling coefficient, A, is whether it should be considered as a mass or volume dependent term. Physics tells us that the specific heat capacity defines the energy required to raise the temperature of $1 \mathrm{~kg}$ of material by $1^{\circ} \mathrm{K}$, illustrating the clear reason for allocating a mass dependency over volume dependency.

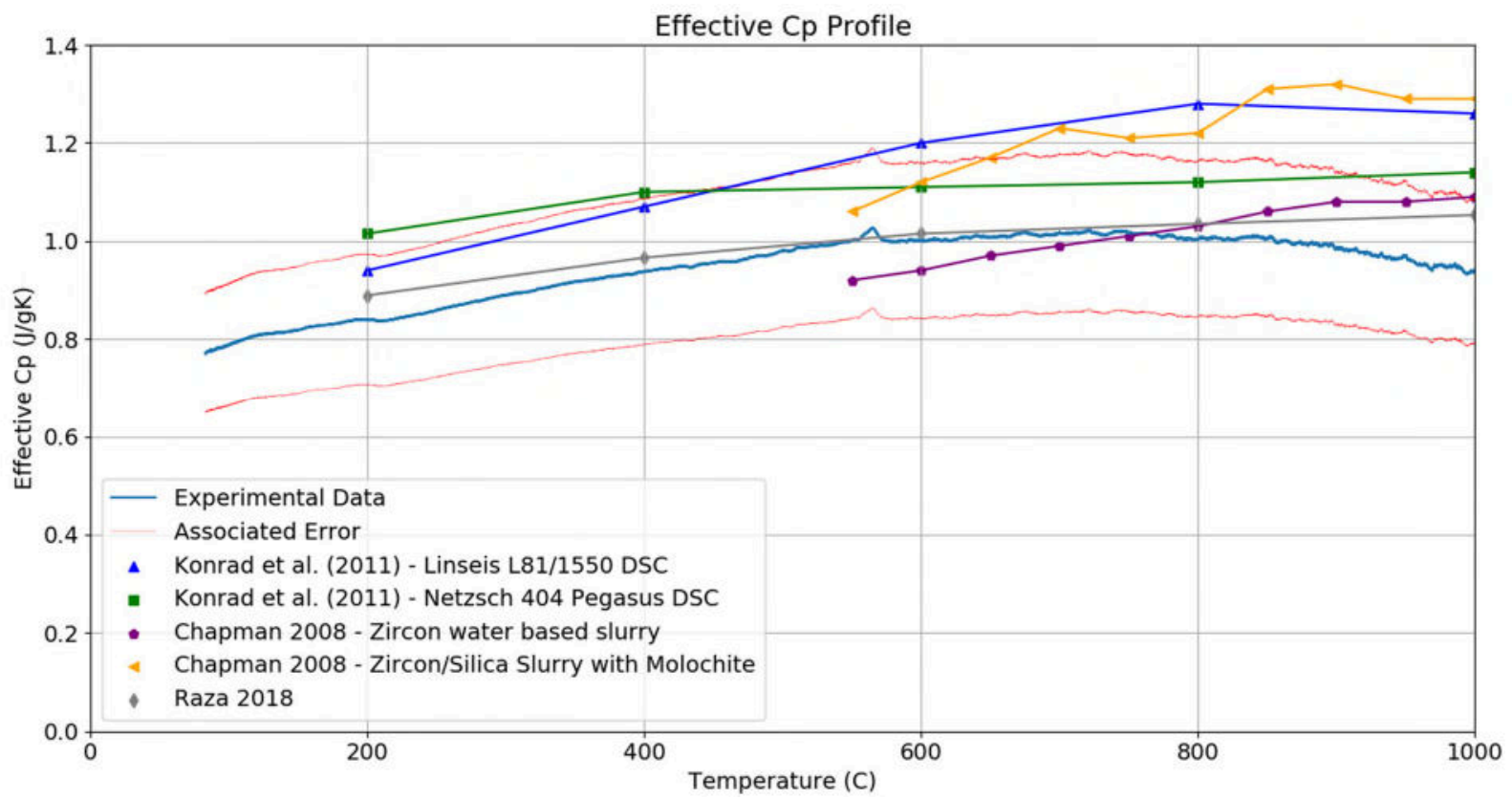

Figure 2. Comparative plot between the experimental $\mathrm{Cp}$ data and previous results from the literature as a function of temperature

Figure 2 shows the results of the specific heat capacity measurements including the calculated scaling factors of the three layers; $A_{1}=0.0548, A_{2}=0.9273$ and $A_{3}=0.0179$ respectively. These factors were evaluated by determining the mass of like-layers relative to the total mass of the sample. Given the output volume of data points a region of uncertainty, bounded by the lines in red, was selected as opposed to presenting an individual error at each point.

Plotted alongside the experimental results are published data from [8] and [10]. The comparison between the results and published data, particularly with the trend of the [10] (Netzsch instrument) and [6], are in very good agreement. In the temperature region approaching $1000^{\circ} \mathrm{C}$, the results of $C_{p}^{e f f}$ begin to drop relative to the other data sets, with the exception of [10] (Linseis instrument) data which plateaus and begins to fall in a similar manner. Such a trend was not expected and may well be caused as a result of the technique for modelling heat capacity.

Our experimental results generally form an uninterrupted curve comparable to those seen in the literature, however a pronounce peak emerged in the profile at $\approx 150^{\circ} \mathrm{C}$. Although the weighting factors have eliminated this peak, they have revealed a second, much smaller peak which is visible in figure 2 at $\approx 550^{\circ} \mathrm{C}$. Peaks that appearing in a DSC profile can be directly attributed to chemical reactions/phase changes that occur between mould components. This remaining peak at $\approx 550^{\circ} \mathrm{C}$ is most likely the result of transitions between polymorphs of Silica, specifically between $\alpha$-quartz and $\beta$-quartz at $573^{\circ} \mathrm{C}$ [11]. The suppressed peak at the lower temperature is also more than likely attributable to Silica as transitions are possible at a temperature a low as $117^{\circ} \mathrm{C}$ [11]. Due to the slow speed of the temperature changes during the experiment, this phase transformation is fully reversible; hence why the lower temperature peak appeared in both the increasing and decreasing cycles of temperature. 


\subsection{Dilatometry}

As both a $12 \mathrm{~mm}$ and a $25 \mathrm{~mm}$ sample were investigated during a heating and cooling phase, four profiles of fractional length change against temperature are available to consider. As indicated by equation 2 ,

$$
\frac{d L}{L_{0}}=\alpha(\Delta T)
$$

where $d L$ is the sample change in length, $L_{0}$ is the original sample length, $\alpha$ is the linear coefficient of thermal expansion and $\Delta T$ is the change in temperature, the CTE can be determined by taking the gradient of these profiles. This was performed for all four profiles and a statistical assessment was conducted to assess the extent to which these data sets represent a statistically significant result. SPSS software was used to perform a linear regression and an analysis of variance (ANOVA) test on each data set and the $\mathrm{R}, \mathrm{R}^{2}$ and $\mathrm{p}$ values respectively were recorded. These tests reveal that of the four available data sets only the $12 \mathrm{~mm}$ sample during the increasing temperature cycle has a statistically significant correlation.

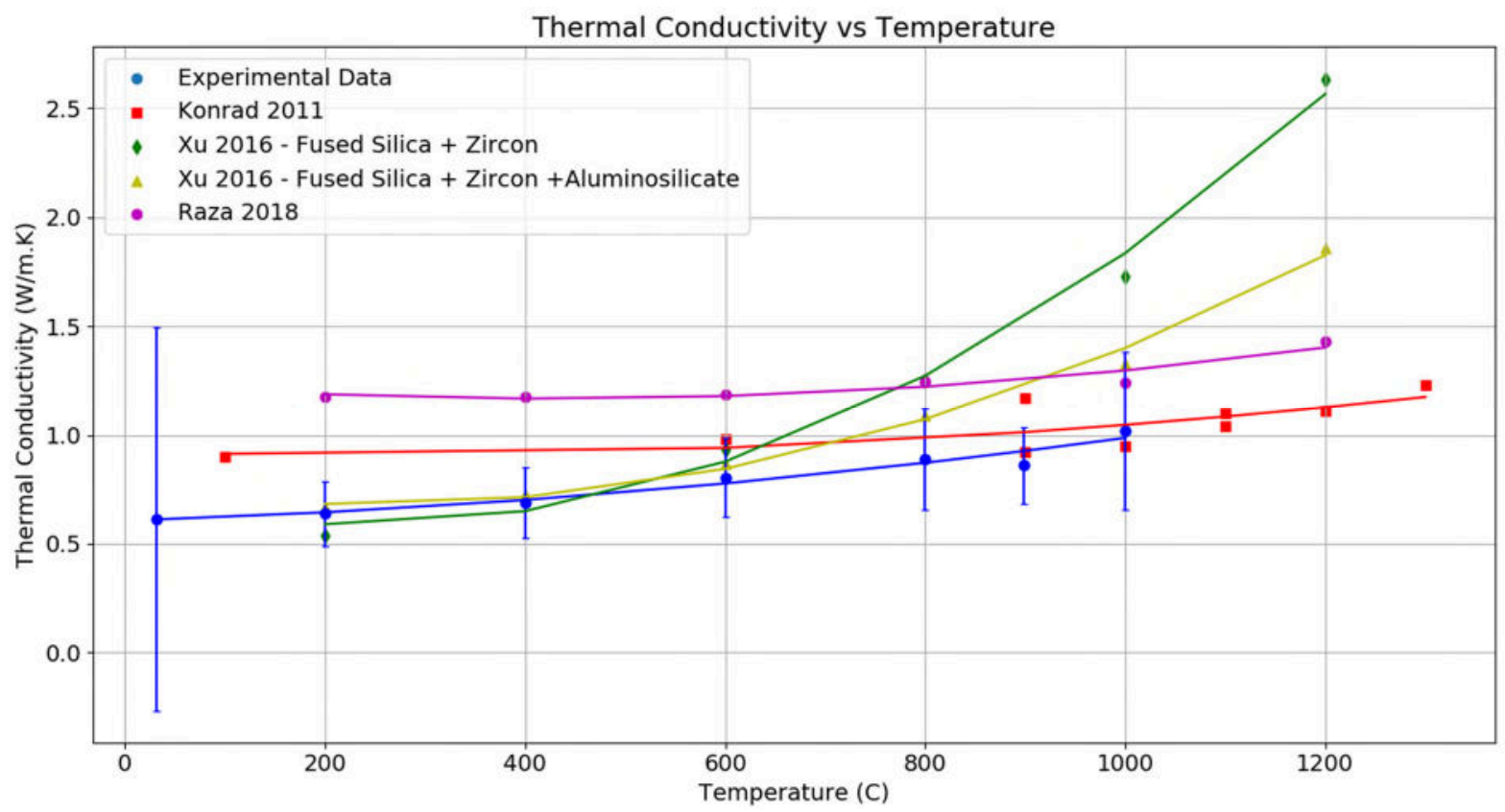

Figure 3. Comparative plot between the calculated thermal conductivity and thermal conductivity data published in literature sources as a function of temperature

Taking gradients of these results has a high sensitivity to noise in the experimental setup if conducted across narrow ranges in temperature. This is due to the fact that large fluctuations are present within very narrow ranges. Performing the assessment across a wider temperature range generates results that are both visually and statistically more in keeping with the expected linear fitting expected from equation 2.

The uncertainty in the CTE measurement using directly comparable equipment has been discussed in the previously published literature [12]. From this work, the uncertainty was determined using the Guide to the Expression of Uncertainty in Measurement to have a value of $5.8 \times 10^{-8} \mathrm{~K}^{-1}$ independent of the measured value.

\subsection{Density and Porosity}

The resulting density determined from the Pycnometry experiment has a value of $2.536 \pm 0.002 \mathrm{~g} / \mathrm{cm}^{3}$ and a porosity value a $60.568 \%$. Although not identical in composition, density measurements quoted in literature [13] for similar shell materials have a lower value; $1.93 \mathrm{~g} / \mathrm{cm}^{3}$ for Alumina-Silica mix and $1.64 \mathrm{~g} / \mathrm{cm}^{3}$ for Zircon-Silica mix. The difference in density can be explained by the use of Zirconia in this study over Zircon in [13]. Zirconia has a higher density relative to Zircon, resulting in a higher overall shell density. 
The porosity value is significantly higher than a $31.7 \%$ porosity value quoted by [13]. Unlike this source however, the porosity of the shell under consideration in this work increases during firing as the fibre reinforcements burn away. As a result, it is not necessarily surprising that the porosity value is so much higher than those of other studies.

\subsection{Thermal Conductivity}

Based on measurements of density, heat capacity and thermal diffusivity, it was possible to calculate the thermal conductivity, shown graphically in figure 3, of the material using equation 3 [14].

$$
\lambda=\rho C_{P} \alpha
$$

Here $\lambda$ is the thermal conductivity, $\rho$ is the density, $C_{P}$ is the heat capacity and $\alpha$ is the thermal diffusivity. As a direct consequence of the limited results from the LFA, thermal conductivity is limited to the same $1000^{\circ} \mathrm{C}$ temperature value. Included in figure 3 are data for similar shell materials gathered from previously published literature [10][14]. Despite an exceptional agreement between our experimental Cp results and other data from [6], the calculated thermal conductivity results fall below those seen in [6] except for the high temperature measurement, which is not necessarily reliable (as a result of LFA complications). Generally, the calculated data is in good agreement with the collection of results from the literature with an average value of $0.789 \mathrm{~W} / \mathrm{m}^{2}$ and conforms to the same trend of increasing with temperature.

\section{$\underline{\text { 4.0 Computational Verifications }}$}

Commercial computer software, specifically Flow-3D®, was employed to verify the experiment results. Unfortunately, as it has not yet been possible to organise a full-scale casting experiment as of yet, there is no possibility of making comparisons between experimental and simulation result. However, as the primary purpose of this characterization work is to inform simulation boundary conditions, aspects of the configuration for such simulations that have been completed are worthy of discussion.

\section{1. - Mesh analysis}

The sensitivity of simulations to the mesh that is established around the geometry is an important consideration initially due to the dramatic effects it can have on computational times and accuracy; this is particularly true when dealing with thin casting cross-sections. Previous literature surrounding mesh analysis [15] indicates that an examination of both the mesh element dimensions and the time-step is necessary to consider a simulation sufficiently optimized.

After conducting trials, it was concluded that a dual mesh configuration was the most appropriate where a coarse mesh was placed over the downsprue \& ingates and a fine mesh placed over the thin section of the component. The final dimensions of the coarse and fine meshes were $1 \mathrm{~mm} \times 1 \mathrm{~mm} \times 1 \mathrm{~mm}$ and $0.5 \mathrm{~mm} \times 0.5 \mathrm{~mm} \times 1 \mathrm{~mm}$ respectively with a time-step of $1 \times 10^{-5} \mathrm{~s}$.

\subsection{Conclusion}

With the appropriate use of scientific instrumentation, it has been possible to determine a comprehensive set of thermophysical properties for an industrial investment casting shell.

These techniques are however not necessarily infallible during implement with factors including the interaction between shell and equipment having a profound effect on the results. Although not all issues have been overcome, values of the linear coefficient of thermal expansion, density, porosity, thermal diffusivity and thermal conductivity were determined as a function of temperature. These values are fundamental foundations for building accurate computer simulations to assess and ideally reduce defects in cast components.

Future work will include comparisons between experimentally obtained data and Flow-3D® computer simulations to verify the findings. In addition, further investigations are intended on the LFA to determine values of the thermal conductivity as a function of temperature in the range above $1000^{\circ} \mathrm{C}$. 


\section{Acknowledgements}

The authors wish to thank the Engineering and Physical Sciences Research Council (EPSRC) [Grant EP/L016389/1] and TPC Components AB for providing the necessary funding for this research.

The authors wish to thank Cranfield University, Jonkoping University and the University of Warwick for providing access to scientific equipment need to complete these investigations.

The authors wish to specifically thank Dr Jacob Steggo and Jörgen Eriksson for their outstanding support during this research.

The data used in this paper is described in CORD at http://doi.org/10.17862/cranfield.rd.9934280

\section{Bibliography}

[1] M. Jolly, "Castings," in Comprehensive Structural Integrity, B. Karihaloo, Ed. Oxford: Elsevier, 2003, pp. 377-466.

[2] E. Kuljanic, Ed., Advanced Maufacturing Systems and Technology. Springer Wien New York, 2005.

[3] M. J. Hendricks, P. M. J. Wang, R. A. Filbrun, and D. K. Well, "Hot MOR and Creep Properties of Common Ceramic Shell Refractories," in 46th Annual Technical Meeting of the Investment Casting Institute, 1998.

[4] C. M. Wolfe, K. L. Holubik, M. J. Hendricks, and P. M. J. Wang, "How Ceramic Shell Properties Predict Resistance to Shell Cracking," pp. 1-16, 2010.

[5] M. J. Hendricks, "An Analysis of Ceramic Shell Materials," in 21st European Conference on Investment Casting, 1990.

[6] M. Raza, "DEVELOPING PROCESS DESIGN METHODOLOGY FOR INVESTMENT CAST THINWALLED STRUCTURES,” Malardalen University, 2018.

[7] J. Gebelin and M. R. Jolly, “"Modelling Filters in Investment Casting,"” in FOCAST 2nd Mini Conference, 2001.

[8] L. A. Chapman et al., "Properties of Alloys and Moulds Relevant to Investment Casting," 2008.

[9] S. Jones, "Summary of Standard Shell Properties Obtained for Inclusion in Modelling Development," in FOCAST 1st Mini Conference, 2000.

[10] C. H. Konrad, M. Brunner, K. Kyrgyzbaev, R. Völkl, and U. Glatzel, "Determination of heat transfer coefficient and ceramic mold material parameters for alloy IN738LC investment castings," J. Mater. Process. Technol., vol. 211, no. 2, pp. 181-186, 2011.

[11] S. Gornostayev, O. Kerkkonen, and J. J. Harkki, "Importance of Mineralogical Data for Influencing Properties of Coke," Process Metall., pp. 770-773, 2006.

[12] T. Matsushita, E. Ghassemali, A. Saro, L. Elmquist, and A. Jarfors, "On Thermal Expansion and Density of CGI and SGI Cast Irons," Metals (Basel)., vol. 5, no. 2, pp. 1000-1019, 2015.

[13] M. Xu, "Characterization of investment shell thermal properties," 2015.

[14] M. Xu, S. N. Lekakh, and L. Von Richards, "Thermal property database for investment casting shells," Int. J. Met., vol. 10, no. 3, pp. 342-347, 2016.

[15] M. Pohanka, K. A. Woodbury, and J. Woolley, "Obtaining Temperature-Dependent Thermal Properties of Investment Casting Mold," in ASME International Mechanical Engineering Congress \& Exposition, 2002 . 


\section{An experimental characterization of} thermophysical properties of a porous ceramic shell used in the investment casting process

Jones, Christopher A.

Springer

Jones CA, Jolly MR, Jarfors AEW, Irwin M. (2020) An experimental characterization of thermophysical properties of a porous ceramic shell used in the investment casting process. In:

TMS 2020 149th Annual Meeting and Exhibition, 23-27 February 2020, San Diego, California, USA https://doi.org/10.1007/978-3-030-36296-6_102

Downloaded from Cranfield Library Services E-Repository 